\title{
Memory Training and Task Specificity in 90- - 99-Year-Old Seniors with Mild Cognitive Impairment
}

\author{
Peter A. Magaro, Bruce Brotter, Mariyam Jalees \\ Memory Training Centers of America, New York, USA \\ Email: petermagaro@memorytrainingcenters.com
}

Received 1 November 2014; revised 21 November 2014; accepted 6 December 2014

Copyright (C 2015 by authors and Scientific Research Publishing Inc.

This work is licensed under the Creative Commons Attribution International License (CC BY). http://creativecommons.org/licenses/by/4.0/

(c) (i) Open Access

\begin{abstract}
Managing memory deficits is a central problem among older adults with mild cognitive impairment (MCI). This study examined the effects of memory training on memory performance in an understudied "oldest-old" population ranging in age from 90 to 99 years. Eighteen mild to moderately cognitive-impaired older seniors, 90 years and older were recruited from memory clinics established in senior living communities. Treatment sessions took place, on average, twice weekly, for 55 minutes. Memory intervention included nineteen computer-based exercises customized to focus on memory loss. The specificity of memory training was very clear; memory training produced significant effects $(F(3,51)=2.81, p=0.05)$ on memory performance, especially after 6 months of training, while other outcome measures showed no effects as predicted. Based on the results, it can be concluded that interventions targeting cognition and memory in the oldest-old MCI population can significantly improve memory function and reduce cognitive deficits.
\end{abstract}

\section{Keywords}

Memory, Cognitive, Training, Cognition, Seniors, Aging, Mild Cognitive Impairment, MCI

\section{Introduction}

There seems to be an agreement that cognitive intervention with older adults is effective in maintaining and even improving cognitive performance and behavior [1]-[7]. This work is impressive because a minimal intervention improves performance on tasks far removed from the memory intervention [8]-[12]. However, even with such robust findings it is not clear what the attributes of the intervention that produce changes are or how specific the change is. For instance, it is not clear if an intervention in one cognitive domain, an area of knowledge or activ- 
ity, "generalizes" to improvements in other domains with or without gains in the domain of intervention (e.g., generalization) or if gains in cognition or behavior are restricted to the domain of the intervention (e.g., intervention specificity). Such considerations have practical implications. For example, it is not known whether generalization or intervention specificity results in greater gains in cognition/behavior for a given cognitive domain, such as memory. It is also not clear if gains in domains other than the one involved in the intervention can be retained for long periods of time. Finally, increasing cognition in a given domain, such as memory, is difficult, as a given intervention may unpredictably produce gains in other unrelated domains without gains in the targeted domain.

Some studies suggest that speed of processing interventions can robustly improve cognition across several domains, therefore demonstrating generalization. But that the benefits to cognitive processes other than the one for which the intervention is targeted may not be retained over time [13]. Smith's [5] "plasticity-based adaptive cognitive training" (IMPACT) study extensively measured memory and attention by many tasks, including the memory subtests of the Repeatable Battery for the Assessment of the Neuropsychological Status (RBANS). The cognitive intervention of interest was speed of processing, because speed of processing was considered a basic process, more so than memory, and showed a natural decline with age. It was also of interest to know whether training on one cognitive process would carry over into improvements in memory functioning. It was concluded that the speed of processing intervention affected all outcome measures, indicating that this training produced gains that generalized to untrained measures of memory and attention, and that these gains were significantly larger than those seen with a program of general cognitive stimulation. A follow-up study [13] found the intervention effect most evident at 3 months after completing the intervention, but the secondary cognitive domains, such as memory, did not retain the benefit.

The "Advanced Cognitive Training for Independent and Vital Elderly" (ACTIVE) studies with neurologically and cognitively intact seniors directly addressed the question of the effectiveness of memory training and speed of processing [8]-[11]. Willis et al. [12] separated speed of processing and memory interventions, and added reasoning as an intervention in adults aged 65 or older. It was found that each intervention (e.g., speed of processing, memory and reasoning) maintained effects on its respective specified targeted cognitive ability through 5 years. Also, "booster training" had a considerable effect on the performance-based functional measures for these cognitive domains. Rebok et al. [10] used the similar 3 interventions and population age and reported improvement in all 3 groups (e.g., speed of processing, memory and reasoning) on the respective specific measures [12]. This improvement lasted for 10 years with a refresher course at 11 and 35 months, but only for reasoning and speed of processing. Ball et al. [11] suggested that the ACTIVE results may indicate that memory training is less effective than other interventions, given the differential effects of intervention domains on their respective cognitive processes.

An alternate explanation is that the memory intervention is not so "strong" as compared to the other interventions. In the Willis study [12], memory intervention involved only teaching mnemonic strategies to the participants, who required less cognitive efforts than memorizing material and engaging either recall or working memory. For instance, when working memory is directly manipulated, effects are similar to the speed of processing results. A study done with neurologically and cognitively intact adults, 60 to 80 years old, who were trained on a complex working memory task for 4 to 5 weeks, that improvement occurred in short-term memory and working memory, as well as on an attention task that was not part of the intervention. Unfortunately, the working memory intervention effect on other cognitive measures was not compared to assess the generalization effect, though the increase in attention performance suggested generalization [14]. The present study examines memory training intervention effects across a wide range of cognitive measures. Differential treatment effects of the memory intervention are tested by examining performance across all subtests of a general neurobehavioral test, the Cognistat battery. The hypothesis is that only the memory subtest will show improvement while other subtests will not be affected.

Another goal of this study was to extend the conclusions from the neurologically and cognitively intact senior population of the ACTIVE/IMPACT groups to a subset of adults diagnosed with MCI. Studies involving cognitive rehabilitation in people with MCI follow a similar pattern as those with non-impaired adults [15]. We will extend this pattern to the MCI older senior in terms of task specificity and type of intervention. For example, the results from training programs showed improvements in the global cognitive status and performance of older adults with MCI in both computer-based cognitive training [6] [7] [16] and other non-computerized memory training interventions [4] [17]. Thus, there is a similarity in results between the intact adult studies and the MCI 
work in terms of the positive effects of memory training for studies that employ specific specialized measured interests. Some MCI results (e.g., [4] [17]) show intervention specificity, while in others generalization occurs [15]. The overall effect of improved performance seems to hold that memory is most often improved and that it is training specific but not a differential effect. Here, treatment specificity effects are examined in an MCI population and compared directly to other cognitive measures.

Another purpose of the current study is to extend the effects of treatment specificity to the older MCI patient who has been ignored in past research or combined into a total elderly group including 60 and 70 years old. Specifically, in the current study, individuals aged 90 - 99 (mean age 92.61 years, SD =2.62), were tested and a memory intervention given. Our knowledge is limited about cognitive losses that occur due to MCI, AD and dementia at advanced ages, where its prevalence continues to be the highest among the oldest patients [18]-[20]. However, there is some evidence that cognitive decline is not a linear function of age and that some older adults respond to cognitive training just as the "younger" older adult [21]. Despite the importance of this population for a full understanding of cognition across the lifespan and despite the need this age group may have for cognitive improvement, very little testing of the "oldest-old" has been conducted. This lack of testing certainly reflects, in part, the lack of availability of this population.

Thus, there are three hypotheses offered in the current study. First, it was hypothesized that memory training, if successful, would enhance memory, but not other cognitive measures. The second hypothesis was that the results in this MCI population would replicate those found previously in a non-impaired population. Finally, we expect that results in this oldest-senior population will replicate those found with the usual senior population.

\section{Methods}

\subsection{Participants}

\subsubsection{General Criteria for Inclusion}

Individuals selected were receiving treatment in memory clinics established in senior living communities. The total population of seniors tested in the memory clinics was 432. Individuals were self-referred or referred by either a family-member or senior living community staff-member. Inclusion criteria for the current study were age (90 to 99 at start of testing), presenting complaint of memory loss, a level of cognitive impairment that was determined through cognitive testing to be mild-to-moderate, and having had 3 follow-up cognitive testing reports post-initial evaluation wherein all Cognistat subscales were completed (with the exception of the Construction subscale, see below). This latter criterion was established to ensure a length of time sufficient for testing the effects of the treatment, resulting in approximately 12 months between initial testing and the third follow-up testing (see results for additional information regarding the length of time between testing sessions). Mild-to-moderate memory loss was used as inclusion criteria in order to maintain consistency regarding overall level of impairment at start of treatment. There were 18 individuals who met criteria, ranging in age from 90 to 99, all of whom presented with a complaint of "memory loss".

A clinical and neurological assessment was conducted to support the research criteria of mild-to-moderate cognitive impairment, and to exclude diagnoses of age-related memory loss or dementia. Other exclusion criteria included intellectual deficiency or the presence of severe psychiatric disorder. All participants were assessed utilizing the Cognistat, which is a cognitive screening instrument normed by age that evaluates five domains of cognitive functioning language, construction, memory, calculation, and reasoning abilities and, with independent measures, assesses levels of consciousness, orientation, and attention. The 25 -minute screening test produces a profile of cognitive abilities, rather than one global score [22] [23], and it is designed so that a patient's successful performance in the cognitive domains does not conceal deficits in others. The scoring system calculates values, ranging from 0 to 12, for each cognitive domain [24]. The Cognistat gauges overall cognitive functioning, as well as specificity regarding the areas of cognitive function, include memory function [25]. The good sensitivity and specificity of this test enables placement of the participant into one of the three categories of cognitive impairment at initial assessment, so that inclusion/exclusion criteria could be followed.

All participants presented with normal or corrected visual and/or auditory acuity adequate to participate in the study intervention, and no participants were taking any psychotropic medication known to impair cognition.

\subsubsection{General Participant Characteristics}

Of the 18 individuals meeting criteria, 15 of the participants were female, and 3 were male. Prior to retirement, 
all individuals worked outside of the home in clerical, managerial, or professional occupations, or were business owners. Six subjects (1/3 sample) reported that they were high school graduates, eight reported completion of 2 years college, and four reported that they were college graduates (i.e., 2/3 sample completed some college or were college grads). Fourteen of the 18 subjects reported engaging in physical exercise on a regular basis, generally walking, but including exercise classes, and swimming. All but 2 subjects reported reading the newspaper on a daily basis, reading books or magazines, and/or playing cards, word-games or other cognitively stimulating activities. Only one subject reported the use of any prescribed medication to treat memory loss. Seventeen of the 18 subjects reported that their experience of memory loss interfered with skills of daily living (one subject not rated).

In sum, the participants were all reasonably well-educated (average years of education 14 = high school grad with 2 years college), remained physically and cognitively active, and remained relatively independent regarding physical capacities and capacity for judgment and decision-making. Most all were conscious of the fact that they were experiencing memory loss (see Table 1 for characteristics of the participants).

Table 1. Demographic characteristics of study sample.

\begin{tabular}{|c|c|c|}
\hline Characteristic & $\mathrm{n}$ & $\%$ \\
\hline \multicolumn{3}{|l|}{ Age } \\
\hline $90-93$ & 11 & $61.0 \%$ \\
\hline $94-96$ & 6 & $33.0 \%$ \\
\hline $97-99$ & 1 & $6.0 \%$ \\
\hline \multicolumn{3}{|l|}{ Gender } \\
\hline Female & 15 & $83.0 \%$ \\
\hline Male & 3 & $17.0 \%$ \\
\hline \multicolumn{3}{|l|}{ Education } \\
\hline HS grad & 6 & $33.3 \%$ \\
\hline Some college & 8 & $44.5 \%$ \\
\hline College Graduate & 4 & $22.2 \%$ \\
\hline \multicolumn{3}{|l|}{ Occupation (previous) } \\
\hline Clerical & 7 & $39.0 \%$ \\
\hline Managerial & 4 & $22.0 \%$ \\
\hline Professional & 5 & $28.0 \%$ \\
\hline Business owner & 2 & $11.0 \%$ \\
\hline \multicolumn{3}{|l|}{ Physical activity } \\
\hline Engages in regular exercise & 14 & $78.0 \%$ \\
\hline Does not engage in physical exercise & 4 & $22.0 \%$ \\
\hline \multicolumn{3}{|l|}{ Cognitive activity } \\
\hline Engages in cognitive stimulation such as reading, board games/word games & 16 & $89.0 \%$ \\
\hline Does not engage in cognitive stimulation & 2 & $11.0 \%$ \\
\hline Acknowledgement of Memory Loss and Impact on daily life function & 17 & $94.0 \%$ \\
\hline Information not available & 1 & $6.0 \%$ \\
\hline \multicolumn{3}{|l|}{ Medication to treat Memory loss } \\
\hline Yes & 1 & $6.0 \%$ \\
\hline No & 17 & $94.0 \%$ \\
\hline \multicolumn{3}{|l|}{ Cognitive competence for legal and healthcare decisions } \\
\hline Legally competent & 15 & $83.0 \%$ \\
\hline Not legally competent & 3 & $17.0 \%$ \\
\hline (Family-member or other holds POA) & & \\
\hline
\end{tabular}




\subsection{General Initial Evaluation}

Upon referral for cognitive evaluation, the need for consult and consent from a Power of Attorney was determined by confirmation of this status with the Wellness Office of the community where subject resided. If a Power of Attorney was assigned for the subject due to concern regarding his/her ability to make important decisions on their own, the POA was contacted to provide proper consent for treatment (only 3 participants required Power of Attorney). Patients were seen by a licensed master's level mental health clinician for an Initial Evaluation session, during which registration and consent forms were completed, medical history was gathered, and patients were asked to report and describe their presenting complaint. Patients were then referred to a doctoral level Clinical Psychologist for the administration of Cognitive Testing.

Based on the test results, each participant was examined for three general diagnostic categories: age-related memory loss; dementia; or mild-to-moderate cognitive impairment. No participants fell into the categories of age-related impairment or dementia. All individuals $(n=18)$ presented with memory loss in the diagnostic range of mild-moderate cognitive impairment, and were then referred for the intervention.

\subsection{Treatment/Intervention}

Individuals $(n=18)$ engaged in the development of a treatment plan with their clinician as the starting point of the intervention. The treatment plan specified areas of concern and goals related to their memory loss, and focused on skills of daily living that were compromised by memory loss.

Treatment sessions took place on average, twice weekly, for 55 minutes (see results for additional information). These sessions were carried out by either doctoral level psychologists or master's level mental health clinicians. The treatment is best-described as professionally administered memory training sessions, during which three types of intervention were administered. The primary intervention was a proprietary internet-based program of exercises that was developed to target the patient's capacity for storage of new information (delayed memory) and working memory. Nineteen exercises were categorized as helping the patient with: verbal memory; visual memory; combined verbal and visual memory; auditory memory, and exercises to strengthen the capacity for attention and concentration. The exercises were constructed to have ten levels of difficulty, with consistent rules that allowed the participant to progress (or not) to the next level of difficulty.

The clinician monitored the participant's engagement in the process, and together, they selected exercises that provided an appropriate level of challenge for the participant. The clinician presented the stimuli of the exercises on the computer, and the participant would respond, with responses then entered into the computer by the clinician. Additionally, the clinician was able to regulate the degree of delay or distraction so that the participant experienced an optimal level of cognitive exercise for both working memory and delayed memory. An example of one of the 19 computer exercises was to present the participant with pictures of faces, with names, and then present the faces without names, challenging the participant to remember the correct face/name pairs. At level one there were 2 faces, with significant differences between the faces regarding gender, age and race. At higher exercise levels, the number of faces/names would increase, and the diversity among the faces would decrease.

In addition to the computer-generated exercises, clinicians provided interactive interventions, such as 3- and 4-word retrieval exercises, inquiry regarding recent events (supported by cues to encourage the patient's ability to retrieve information), and other interactive memory-exercises based on the participant's treatment goals or area of interest. Treatment goals and areas of interest were determined by examination of these with each individual patient. For example, a participant who was previously a managing partner in a law firm was provided with an intervention of the clinician reading passages of legal cases, and challenging the participant to recall the information from the passage.

The third type of intervention was to help the participant develop compensatory skills to strengthen independent and confident functioning. Some examples of these practical strategies were: helping the participant to identify a place in their apartment to keep important items (that were otherwise frequently misplaced); use of a calendar; wearing a watch; utilizing appointment cards; carrying a schedule of the day's activities.

Cognitive testing was routinely re-administered, generally on a quarterly basis, though there was some variation in time intervals between testing's, based on patient availability and other scheduling constraints (See Results for additional information). At the time of cognitive testing, the patient's treatment plan was also revised with their treating clinician, to determine the patient's subjective experience of progress, while the testing report provided the objective measure of progress for the study. Here, results from the Cognistat are examined. The test 
was administered during the initial neurological assessment, and so all participants completed the Cognistat 4 times; time 1 being initial evaluation, and times 2, 3, and 4 being those Times following treatment/intervention after approximately 3,6, or 9 months, respectively. Given that the Cognistat and CVLT have their emphasis on different cognitive skills, and given the extensive analyses presented here, results of the CVLT will be reported elsewhere.

\section{Analyses}

\subsection{Results}

Repeated Measures ANOVAs were conducted on raw scaled scores as a function of Cognistat subscale and time. Subscales analyzed were Memory, Orientation, Attention, Communication, Repetition, Naming, Calculation, Similarity, and Judgment. Construction was not included in analyses because 8 individuals did not complete all testing times for this measure (see Table 2 for means and standard deviations as a function of Cognistat subscale and time).

The number of Weeks intervening between times, and the number of Training Sessions between each test time were regressed against performance for each Cognistat subscale as a function of time, as well as cumulatively over time, in a series of multiple regression analyses. Correlations were conducted between average number of training sessions per week as a function of time as well.

In order to i) be able to compare scores between subscales and ii) explicitly examine improvement at subsequent times relative to time 1 (pre intervention), scores were converted to percentage improvement scores relative to time 1 via the formula ((time X divided by time 1) times 100) minus 100). Repeated measures ANOVAs were conducted on these percentage improvement scores as a function of Cognistat subscale and time. Additionally, a series of paired t-tests were conducted comparing performance at each time for each Cognistat subscale (see Table 3 for means and standard deviations as a function of Cognistat subscale and time for these percentage scores). Multiple regression analyses and correlations, similar to those performed for raw scores, were also conducted on percentage improvement scores.

\subsection{Raw Scores}

\subsubsection{Repeated ANOVAS}

The repeated measures ANOVA examining Memory performance raw score as a function of time was significant $(F(3,51)=2.81, p=0.05$. Analyses of simple effects revealed that memory performance at time 4 was significantly greater than performance at time 2 (paired $\mathrm{t}$-test $(\mathrm{t}(17)=2.72, \mathrm{p}=0.01, \mathrm{~d}=0.69$ ). There was a strong trend for time 3 performance to be greater than time 2 as well $(t(17)=1.94, p=0.07)$. Cohen's $d$ for this latter

Table 2. Raw score means and standard deviations as a function of cognistat subscale and time.

\begin{tabular}{ccccc}
\hline & \multicolumn{3}{c}{ Raw score means and standard deviation as a function of Cognistat subscale and time } \\
\hline & \multicolumn{3}{c}{ Time } \\
Subscale & 1 & 2 & 3 & 4 \\
Memory & $4.278(1.78)$ & $3.61(2.09)$ & $4.44(2.18)$ & $5.17(2.38)$ \\
Orientation & $7.44(3.71)$ & $7.83(3.62)$ & $7.89(3.58)$ & $7.72(3.64)$ \\
Attention & $6.94(1.86)$ & $7.11(1.08)$ & $6.94(1.39)$ & $6.94(1.26)$ \\
Comprehension & $5.61(0.61)$ & $5.44(0.70)$ & $5.44(0.98)$ & $5.89(1.97)$ \\
Repetition & $10.56(2.01)$ & $11.72(1.07)$ & $11.56(1.20)$ & $11.11(1.28)$ \\
Naming & $7.78(1.52)$ & $7.94(1.39)$ & $7.61(1.68)$ & $7.39(1.85)$ \\
Calculation & $4.00(0.77)$ & $3.61(0.98)$ & $3.61(0.85)$ & $3.67(1.03)$ \\
Similarity & $6.11(1.45)$ & $6.11(1.32)$ & $5.72(1.41)$ & $5.50(1.30)$ \\
Judgment & $5.06(0.64)$ & $4.78(0.73)$ & $4.67(0.91)$ & $4.83(0.79)$ \\
\hline
\end{tabular}


Table 3. Means and standard deviations of percentage improvement relative to time 1 as a function of cognistat subscale and time.

\begin{tabular}{cccc}
\hline & \multicolumn{2}{c}{$\begin{array}{c}\text { Means and standard deviations of parentage improvement relative to time } 1 \\
\text { as a function of the Cognistat subscale and time }\end{array}$} \\
\hline Subscale & 2 & Time & 4 \\
Memory & $-6.24(71.25)$ & $19.50(77.73)$ & $36.61(85.30)$ \\
Orientation & $15.58(46.57)$ & $16.52(52.17)$ & $11.83(40.78)$ \\
Attention & $12.82(44.85)$ & $8.98(45.37)$ & $8.52(39.71)$ \\
Comprehension & $-2.41(12.46)$ & $-2.13(19.40)$ & $6.11(35.19)$ \\
Repetition & $16.12(32.14)$ & $13.81(28.14)$ & $-5.70(23.34)$ \\
Naming & $2.83(8.72)$ & $0.60(25.14)$ & $-3.43(38.86)$ \\
Calculation & $-8.80(24.00)$ & $-4.82(36.25)$ & $-7.45(21.35)$ \\
Similarity & $3.80(29.29)$ & $-2.78(27.68)$ & $-3.89(13.54)$ \\
Judgment & $-4.63(15.65)$ & $-7.04(17.45)$ & \\
\hline
\end{tabular}

comparison revealed a moderate effect size $(d=0.39)$, suggesting that the lack of traditional significance may reflect the relatively small sample size (Figure 1).

The repeated measures ANOVA examining repetition performance was significant $(F(3,51)=4.58, p<0.01)$. Analyses of simple effects revealed that repetition performance at time 3 was significantly greater than Time 1 (paired t-test $\mathrm{t}(17)=2.26, \mathrm{p}<0.05, \mathrm{~d}=0.60$ ). Performance at time 4 was significantly decreased relative to time $2(\mathrm{t}(17)=2.5, \mathrm{p}<0.05, \mathrm{~d}=0.52)$.

Examination of the other subscales revealed no other significant effects ( $p>0.20$ for all comparisons) (see Table 3 for means and standard deviations of raw scores as a function of Cognistat subscale and time).

\subsubsection{Multiple Regressions}

The number of intervening weeks and number of intervening training sessions from time 1 to time 2 were regressed against each Cognistat subscale time 2 score (see Table 4 for the average number of intervening weeks and sessions between each time). The regression against orientation approached significance $\left(\mathrm{R}_{2}=0.32, \mathrm{~F}(2,15)\right.$ $=3.59, \mathrm{p}=0.053)$, with number of intervening training sessions negatively predicting orientation performance at time $2(\beta=-0.56, \mathrm{t}=-2.60, \mathrm{p}<0.05)$. The number of intervening weeks and number of intervening training sessions from time 2 to time 3 were regressed against each Cognistat subscale time 3 score. The regression against similarities was significant $\left(\mathrm{R}_{2}=0.38, \mathrm{~F}(2,15)=4.53, \mathrm{p}<0.05\right)$, with number of intervening training sessions negatively predicting Similar performance at time $3(\beta=-0.79, \mathrm{t}=-2.86, \mathrm{p}=0.01)$. The number of intervening weeks and number of intervening training sessions from time 3 to time 4 were regressed against each Cognistat subscale time 4 score. There were no significant relationships. The cumulative number of intervening weeks and the cumulative number of intervening training sessions from times 1 to 3 were regressed against each Cognistat subscale time 3 score. The regression against similar performance approached significant $\left(\mathrm{R}_{2}=0.38\right.$, $\mathrm{F}(2,15)=3.47, \mathrm{p}=0.058$ ), with performance on the similar subscale negatively predicted by the cumulative number of intervening training sessions between times 1 and $3(\beta=-0.48, \mathrm{t}=-2.63, \mathrm{p}<0.05)$. The cumulative number of intervening weeks and the cumulative number of intervening training sessions from times 1 to 4 were regressed against each Cognistat subscale time 4 score. The regression against repetition was significant $\left(\mathrm{R}_{2}=\right.$ $0.44, \mathrm{~F}(2,15)=6.02, \mathrm{p}=0.01$ ), with number of intervening weeks negatively predicting repetition performance at Time $4(\beta=-0.67, \mathrm{t}=-3.46, \mathrm{p}<0.01)$.

\subsubsection{Correlations}

The average number of training sessions per week as a function of time was calculated via the formula (number of training sessions between times $\mathrm{X}$ and $\mathrm{Y}$ divided by number of weeks intervening between times $\mathrm{X}$ and $\mathrm{Y}$ ). 
Mean (SD) Raw Score on the Memory Subscale of the

Cognistat as a Function of Times 1, 2, 3, and 4

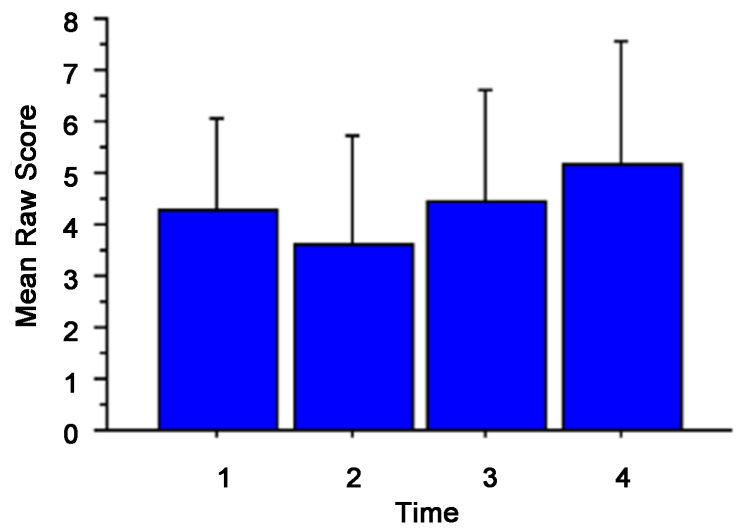

Figure 1. Mean (SD) raw score on the memory subscale of the Cognistat as a function of time $1,2,3$, and 4 .

Table 4. Number of intervening weeks and training sessions as a function of test time (SD).

\begin{tabular}{|c|c|c|c|c|c|}
\hline \multirow[b]{2}{*}{$\begin{array}{l}\text { Number of intervening } \\
\text { Weeks/training sessions }\end{array}$} & \multicolumn{4}{|c|}{$\begin{array}{l}\text { Number of intervening weeks and training sessions } \\
\text { as a function of test time (SD) intervening time }\end{array}$} & \multirow[b]{2}{*}{1 to 4} \\
\hline & 1 to 2 & 2 to 3 & 3 to 4 & 1 to 3 & \\
\hline Weeks & $17.17(6.71)$ & $16.61(5.23)$ & $17.00(4.13)$ & $32.78(8.69)$ & $49.78(11.12)$ \\
\hline Training sessions & $15.39(7.38)$ & $18.72(10.37)$ & $21.00(10.40)$ & $34.11(14.20)$ & $55.11(22.54)$ \\
\hline $\begin{array}{l}\text { Average number of training Ses- } \\
\text { sions per week }\end{array}$ & $1.00(0.50)$ & $1.21(50)$ & $1.24(0.48)$ & $1.10(0.49)$ & $1.14(0.47)$ \\
\hline
\end{tabular}

Correlations between average number of training sessions per week and score on each Cognistat subscale as a function of time were conducted. The average number of training sessions per week between times 1 and 2 was negatively correlated with performance on the orientation subscale $(r=-0.48, p<0.05)$. Average number of training sessions per week between times 2 and 3 negatively correlated with performance on the similar subscale $(\mathrm{r}=-0.60, \mathrm{p}<0.01)$. There was no relationship between the average number of training sessions per week between times 3 and 4 and any Cognistat subscale. The average number of training sessions per week between times 1 and 3 was negatively correlated with performance on the similar subscale of the Cognistat $(r=-0.46, p$ $=0.054)$. The average number of training sessions per week between times 1 and 4 was not correlated with any subscale performance.

\subsubsection{Percentage Improvement Scores}

The repeated measures ANOVA examining improvement relative to time 1 on the Memory subscale was significant $(\mathrm{F}(2,34)=3.84, \mathrm{p}<0.05)$. Analyses of simple effects revealed that improvement in memory performance at Time 4 was significantly greater than improvement in performance at time 2 (paired t-test $t(17)=2.45, p<$ $0.05, \mathrm{~d}=0.54$; Figure 2).

The repeated measures ANOVA examining repetition performance was significant $(F(2,34)=3.33, p<0.05$. Analyses of simple effects revealed that repetition performance at time 4 was significantly less than time 2 (paired t-test $\mathrm{t}(17)=2.32, \mathrm{p}<0.05, \mathrm{~d}=0.60)$. Performance at time 4 was significantly decreased relative to time $2(t(17)=2.5, p<0.05, d=0.26)$. Examination of the other subscales revealed no other significant effects $(p>$ 0.20 for all comparisons) (see Table 4 for means and standard deviations of percentage improvement scores as a function of Cognistat subscale and time).

Percentage improvement scores for each Cognistat subscale as a function of time were analyzed via three (times 2, 3, and 4) repeated measures ANOVAs. There were no significant differences between Cognistat subscales in percentage scores for times 2 or 3 . The ANOVA for time 4 was significant $(F(8,136)=2.071, p<0.05$; Figure 3). Analyses of simple effects via a series of paired t-tests revealed memory percentage improvement 


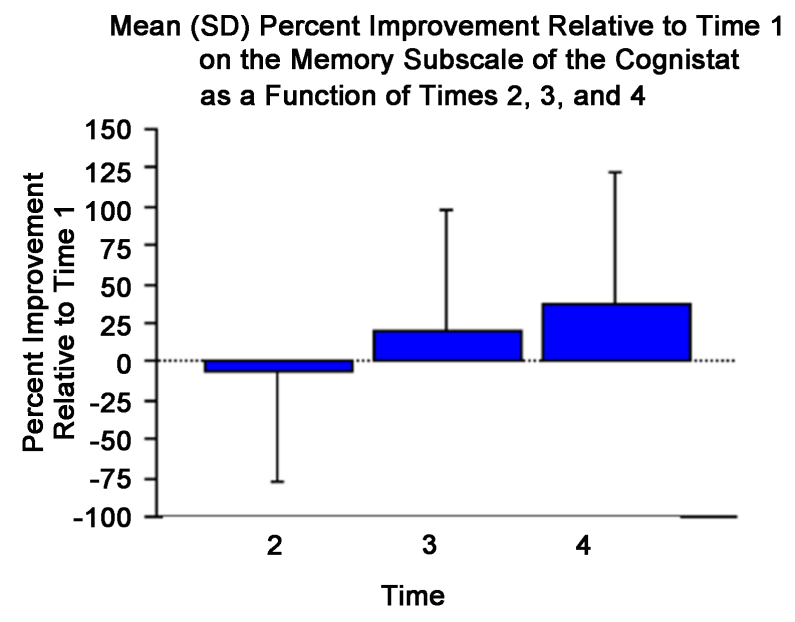

Figure 2. Mean (SD) percent improvement relative to time 1 on the memory subscale of the Cognistat as a function of times 2, 3, and 4 .

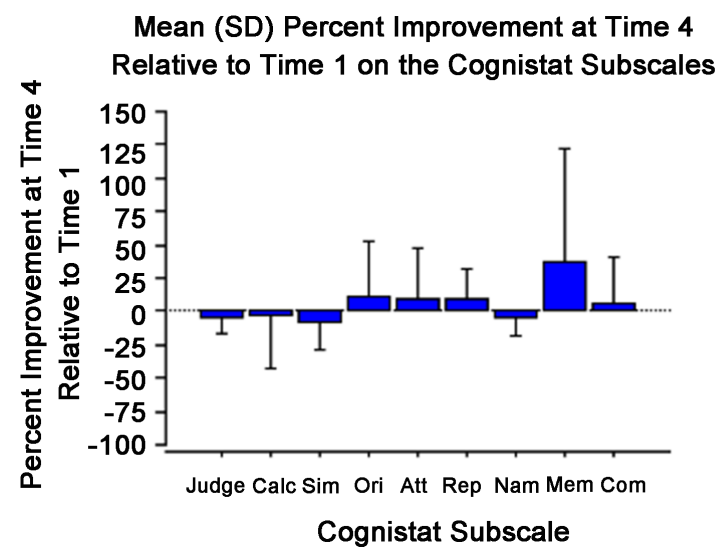

Figure 3. Mean (SD) percent improvement at time 4 relative to time 1 on the Cognistat Subscales.

was significantly greater than that of similarity $(\mathrm{t}(17)=44.07, \mathrm{p}<0.05)$ and naming $(\mathrm{t}(17)=41.94, \mathrm{p}<0.05)$, and trending toward significantly greater than judgment $(\mathrm{t}(17)=40.50, \mathrm{p}=0.055)$. Repetition was tending toward significantly greater than judgment $(\mathrm{t}(17)=12.58, \mathrm{p}=0.059)$. No other comparisons reached significance.

\subsubsection{Multiple Regressions}

The number of intervening weeks and number of intervening training sessions from time 2 to time 3 were regressed against each percentage improvement score as a function of each Cognistat subscale at time 3 . The only significant result was that of calculation $\left(\mathrm{R}_{2}=0.34, \mathrm{~F}(2,15)=3.87, \mathrm{p}<0.04\right)$, however the predictive value of neither variable reached significance ( $p>0.10$ for both variables). The number of intervening weeks and number of intervening training sessions from time 3 to Time 4 were regressed against each percentage improvement score as a function of each Cognistat subscale at time 4 . Results revealed a significant relationship $\left(R_{2}=0.42\right.$, $\mathrm{F}(2,15)=5.40, \mathrm{p}<0.05)$ for the orientation subscale, with number of intervening training sessions positively predicting improvement on orientation $(\beta=0.66, \mathrm{t}=2.92, \mathrm{p}=0.01)$. Naming also was predicted $\left(\mathrm{R}_{2}=0.40\right.$, $\mathrm{F}(2,15)=5.10, \mathrm{p}<0.05)$ by intervening sessions $(\beta=0.50, \mathrm{t}=2.17, \mathrm{p}<0.05)$ and negatively by intervening weeks $(\beta=-0.71, \mathrm{t}=-3.11, \mathrm{p}<0.01)$.

The cumulative number of intervening weeks and the cumulative number of intervening training sessions from times 1 to 3 were regressed against percentage Improvement score as a function of each Cognistat subscale at time 3. There were no significant relationships. The cumulative number of intervening weeks and the cumulative number of intervening training sessions from times 1 to 4 were regressed against percentage improvement 
score as a function of each Cognistat subscale at time 4 . The only moderately significant relationship was that between orientation improvement and number of intervening training sessions $(\beta=0.49, \mathrm{t}=2.16, \mathrm{p}<0.05)$, though the overall regression was not significant $\left(\mathrm{R}_{2}=0.26, \mathrm{~F}(2,15)=2.69, \mathrm{p}=0.10\right)$.

\subsubsection{Correlations}

Correlations between average number of training sessions per week and percentage improvement score on each Cognistat subscale as a function of times 2, 3, and 4, as well as cumulatively between times 1 and 3 and 1 and 4 , were conducted. Percentage improvement in orientation at time 4 was positively correlated with number of sessions/week between times 3 to $4(\mathrm{r}=0.50, \mathrm{p}<0.05)$. No other relationships reached significance.

\section{Discussion}

The current results support prior work showing the specificity of memory training on cognitive performance. Our first hypothesis was confirmed because only a memory task showed positive change after memory training while other tasks did not. The improvement in memory and no other cognitive function is important because it demonstrates that the memory intervention used here may be a valuable tool to manage memory deficits. The fact that this effect is most effective after 6 months of training and then level-off suggests that 6 months may be an appropriate length of time required for this treatment to be effective. There are limitations with such conclusions mainly the small size of the sample and the fact that we were employing treatment protocols as our data base although no tester had any awareness of any investigation of testing variables. The improvement in memory and in no other cognitive functions is important because it indicates that factors other than the intervention itself (e.g., contact with tester or treater generally) do not produce a general increase in cognition, and supports the notion that it is the memory intervention, including the memory task interactions, that is improving memory. Indeed, performance on the repetition subscale actually decreased over the testing time, in contrast to the significant increase in memory performance, indicating that mere exposure to the testing situation did not improve cognition. The fact that memory performance at time 4 is significant or nearly so better than that of three other subscales further indicates that memory improvement is specifically enhanced here.

There was no relationship between memory performance and the number of intervening training sessions or weeks between testing. We expected improvement in memory to be related to the number of training sessions. Number of intervening weeks could be predicted to be negatively related to Cognistat performance, particularly at the longest time since initial testing (e.g., 9 months post the start of the testing), if cognition was declining generally with age. Future research can address this issue. We do know that the optimum time in treatment is 6 months, but it does not seem to be important if 20 or 30 sessions are necessary to reach 6 months.

Results with the oldest MCI population support our second hypothesis and replicate those found previously for a non-impaired population and a younger MCI population. Since MCI captures a group of individuals in a transitional state between normal aging and $\mathrm{AD}$, it is critical to understand that their symptoms can be potentially improved by cognitive memory training and that certain neural patterns possibly linked to particular individual activation patterns can be elicited and strengthened through specific interventions. The present results suggest a method to localize brain changes with functional tasks implications for reductions in $\mathrm{AD}$ (for example, extension in time to $\mathrm{AD}$ diagnosis or severe memory loss) subsequent to memory improvement using the current memory training program deserve further investigation.

Extending the effects of treatment specificity to an understudied "oldest-old" MCI population as stated in our third hypothesis offers some of the first support that cognition can be enhanced in this oldest-old population using a training program. Such results indicate that older adults can be treated successfully contrary to reports that learning in the older senior is questionable [26]. That memory function can be improved in such a population offers insight into cognition generally, and indicates that memory decline in old age is neither inevitable nor irreversible. From a neurophysiological perspective, it may be that neuronal plasticity in the oldest-old is greater than thought and that this neuronal plasticity may be taken advantage of in order to produce gains in function. The brains of people who experience cognitive decline remain highly plastic [1] [27] and training-related brain changes can involve the activation of new alternative brain areas that are not recruited during the memory task prior to training [27]. Evidence from previous studies suggests that daily computer training aimed to stimulate specific cognitive areas has a possibility to individualize treatment on the basis of the patient's neuropsychological pattern, in order to stimulate the damaged areas [3]. An interesting finding [28] reports that training in 
working memory produces neural activity more in short periods of training, but after longer periods the activity decreases which can be interpreted that the neural structure forms the memory connection and does not require the activation to maintain the recall in the short term.

Thus, the memory training that has shown significant improvements in improving cognitive deficits in older people can be used more with neurological interventions to develop guidelines to specify neuropsychological localizations [2]. When speaking of changes in actual brain structure, specific interventions can prove effective in increasing cellular transmission and neural changes that are quantitative with brain imaging [4].

\section{Acknowledgements}

The authors would like to thank Ruth Propper for statistical advising and for assistance with manuscript preparation.

\section{References}

[1] Belleville, S., Clément, F., Mellah, S., Gilbert, B., Fontaine, F. and Gauthier, S. (2011) Training-Related Brain Plasticity in Subjects at Risk of Developing Alzheimer's Disease. Brain: A Journal of Neurology, 134, 1623-1634.

[2] Gunther, V.K., Schafer, P., Holzner, B.J. and Kemmler, G.W. (2003) Long-Term Improvements in Cognitive Performance through Computer-Assisted Cognitive Training: A Pilot Study in a Residential Home for Older People. Aging \& Mental Health, 7, 200-206. http://dx.doi.org/10.1080/1360786031000101175

[3] Peretz, C., Korczyn, A.D., Shatil, E., Aharonson, V., Birnboim, S. and Giladi, N. (2011) Computer-Based, Personalized Cognitive Training versus Classical Computer Games: A Randomized Double-Blind Prospective Trial of Cognitive Stimulation. Neuroepidemiology, 36, 91-99. http://dx.doi.org/10.1159/000323950

[4] Rapp, S., Brenes, G. and Marsh, A.P. (2002) Memory Enhancement Training for Older Adults with Mild Cognitive Impairment: A Preliminary Study. Aging \& Mental Health, 6, 5-11. http://dx.doi.org/10.1080/13607860120101077

[5] Smith, G. (2009) A Cognitive Training Program Based on Principles of Brain Plasticity: Results from the Improvement in Memory with Plasticity-Based Adaptive Cognitive Training (IMPACT) Study. Journal of the American Geriatrics Society, 57, 594-603. http://dx.doi.org/10.1111/j.1532-5415.2008.02167.x

[6] Stavros, Z., Fotini, K. and Magda, T. (2010) Computer Based Cognitive Training for Patients with Mild Cognitive Impairment (MCI). Proceedings of the 3rd International Conference on Pervasive Technologies Related to Assistive Environments, No. 21. http://dx.doi.org/10.1145/1839294.1839319

[7] Talassi, E., Guerreschi, M., Feriani, M., Fedi, V., Bianchetti, A. and Trabucchi, M. (2007) Effectiveness of a Cognitive Rehabilitation Program in Mild Dementia (MD) and Mild Cognitive Impairment (MCI): A Case Control Study. Archives of Gerontology and Geriatrics, 44, 391-399. http://dx.doi.org/10.1016/j.archger.2007.01.055

[8] Wolinsky, F.D., Vander Weg, M.W., Martin, R., Unverzagt, F.W., Willis, S.L., Marsiske, M. and Tennstedt, S.L. (2010) Does Cognitive Training Improve Internal Locus of Control Among Older Adults? Journals of Gerontology Series B: Psychological Sciences and Social Sciences, 65B, 591-598. http://dx.doi.org/10.1093/geronb/gbp117

[9] Wolinsky, F.D., Unverzagt, F.W., Smith, D.M., Jones, R., Stoddard, A. and Tennstedt, S.L. (2006) The ACTIVE Cognitive Training Trial and Health-Related Quality of Life: Protection That Lasts for 5 Years. The Journals of Gerontology: Series A, Biological Sciences and Medical Sciences, 61, 1324-1329. http://dx.doi.org/10.1093/gerona/61.12.1324

[10] Rebok, G.W., Ball, K., Guey, L.T., Jones, R.N., Kim, H., King, J.W. and Willis, S.L. (2014) Ten-Year Effects of the Advanced Cognitive Training for Independent and Vital Elderly Cognitive Training Trial on Cognition and Everyday Functioning in Older Adults. Journal of the American Geriatrics Society, 62, 16-24. http://dx.doi.org/10.1111/jgs.12607

[11] Ball, K., Berch, D.B., Helmers, K.F., Jobe, J.B., Leveck, M.D. and Marsiske, M., the ACTIVE Study Group (2002) Effects of Cognitive Training Interventions with Older Adults: A Randomized Controlled Trial. JAMA: The Journal of the American Medical Association, 288, 2271-2281. http://dx.doi.org/10.1001/jama.288.18.2271

[12] Willis, S.L., Tennstedt, S.L., Marsiske, M., Ball, K., Elias, J. and Koepke, K.M., the ACTIVE Study Group (2006) Long-Term Effects of Cognitive Training on Everyday Functional Outcomes in Older Adults. JAMA: The Journal of the American Medical Association, 296, 2805-2814. http://dx.doi.org/10.1001/jama.296.23.2805

[13] Zelinski, E.M., Spina, L.M., Yaffe, K., Ruff, R., Kennison, R.F., Mahncke, H.W. and Smith, G.E. (2011) Improvement in Memory with Plasticity-Based Adaptive Cognitive Training: Results of the 3-Month Follow-Up. Journal of the American Geriatrics Society, 59, 258-265. http://dx.doi.org/10.1111/j.1532-5415.2010.03277.x

[14] Richmond, L.L., Morrison, A.B., Chein, J.M. and Olson, I.R. (2011) Working Memory Training and Transfer in Older Adults. Psychology and Aging, 26, 813-822. http://dx.doi.org/10.1037/a0023631 
[15] Kinsella, G.J., Mullaly, E., Rand, E., Ong, B., Burton, C., Price, S. and Storey, E. (2009) Early Intervention for Mild Cognitive Impairment: A Randomised Controlled Trial. Journal of Neurology, Neurosurgery, and Psychiatry, 80, 730736. http://dx.doi.org/10.1136/jnnp.2008.148346

[16] Cipriani, G., Bianchetti, A. and Trabucchi, M. (2006) Outcomes of a Computer-Based Cognitive Rehabilitation Program on Alzheimer's Disease Patients Compared with Those on Patients Affected by Mild Cognitive Impairment. Archives of Gerontology and Geriatrics, 43, 327-335. http://dx.doi.org/10.1016/j.archger.2005.12.003

[17] Troyer, A.K., Murphy, K.J., Anderson, N.D., Moscovitch, M. and Craik, F.I.M. (2008) Changing Everyday Memory Behaviour in Amnestic Mild Cognitive Impairment: A Randomised Controlled Trial. Neuropsychological Rehabilitation, 18, 65-88. http://dx.doi.org/10.1080/09602010701409684

[18] Corrada, M.M., Berlau, D.J. and Kawas, C.H. (2012) A Population-Based Clinicopathological Study in the Oldest-Old: The 90+ Study. Current Alzheimer Research, 9, 709-717. http://dx.doi.org/10.2174/156720512801322537

[19] Prohovnik, I., Perl, D.P., Davis, K.L., Libow, L., Lesser, G. and Haroutunian, V. (2006) Dissociation of Neuropathology from Severity of Dementia in Late-Onset Alzheimer Disease. Neurology, 66, 49-55. http://dx.doi.org/10.1212/01.wnl.0000191298.68045.50

[20] Savva, G.M., Wharton, S.B., Ince, P.G., Forster, G., Matthews, F.E. and Brayne, C. (2009) Age, Neuropathology, and Dementia. The New England Journal of Medicine, 360, 2302-2309. http://dx.doi.org/10.1056/NEJMoa0806142

[21] Kawas, C.H. (2008) The Oldest Old and the 90+ Study. Alzheimer's \& Dementia: The Journal of the Alzheimer's Association, 4, S56-S59. http://dx.doi.org/10.1016/j.jalz.2007.11.007

[22] Keirnan, R.J., Mueller, J., Langston, W. and Dyke, C.V. (1987) The Neurobehavioral Cognitive Status Examination: A Brief but Differentiated Approach to Cognitive Assessment. Annals of Internal Medicine, 107, 481-485.

[23] Mueller, J., Kiernan, R. and Langston, W. (2001) Manual for Cognistat (Neurobehavioral Status Exam, NCSE). The Northern California Neurobehavioral Group Inc., Fairfax.

[24] Schwamm, L.H., Van Dyke, C., Kiernan, R.J., Merrin, E.L. and Mueller, J. (1987) The Neurobehavioral Cognitive Status Examination: Comparison with the Cognitive Capacity Screening Examination and the Mini-Mental State Examination in a Neurosurgical Population. Annals of Internal Medicine, 107, 486-491.

[25] Rabin, L.A., Barr, W.B. and Burton, L.A. (2005) Assessment Practices of Clinical Neuropsychologists in the United States and Canada: A Survey of INS, NAN, and APA Division 40 members. Archives of Clinical Neuropsychology, 20, 33-65. http://dx.doi.org/10.1016/i.acn.2004.02.005

[26] Champley, J., Scherz, J.W., Apel, K. and Burda, A.N. (2008) A Preliminary Analysis of Reading Materials and Strategies Used by Older Adults. Communication Disorders Quarterly, 29, 131-140. http://dx.doi.org/10.1177/1525740108315365

[27] Belleville, S., Gilbert, B., Fontaine, F., Gagnon, L., Ménard, É. and Gauthier, S. (2006) Improvement of Episodic Memory in Persons with Mild Cognitive Impairment and Healthy Older Adults: Evidence from a Cognitive Intervention Program. Dementia and Geriatric Cognitive Disorders, 22, 486-499. http://dx.doi.org/10.1159/000096316

[28] Loosli, S.V., Buschkuehl, M., Perrig, W.J. and Jaeggi, S.M. (2012) Working Memory Training Improves Reading Processes in Typically Developing Children. Child Neuropsychology: A Journal on Normal and Abnormal Development in Childhood and Adolescence, 18, 62-78. http://dx.doi.org/10.1080/09297049.2011.575772 
Scientific Research Publishing (SCIRP) is one of the largest Open Access journal publishers. It is currently publishing more than 200 open access, online, peer-reviewed journals covering a wide range of academic disciplines. SCIRP serves the worldwide academic communities and contributes to the progress and application of science with its publication.

Other selected journals from SCIRP are listed as below. Submit your manuscript to us via either submit@scirp.org or Online Submission Portal.
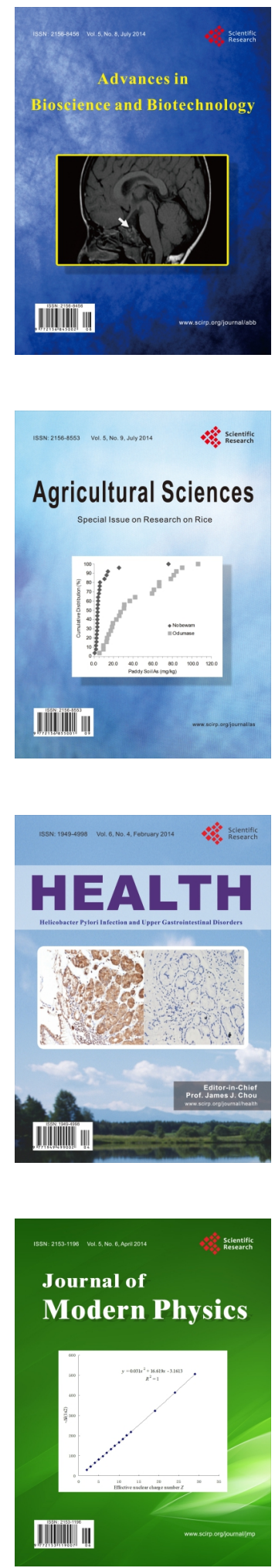
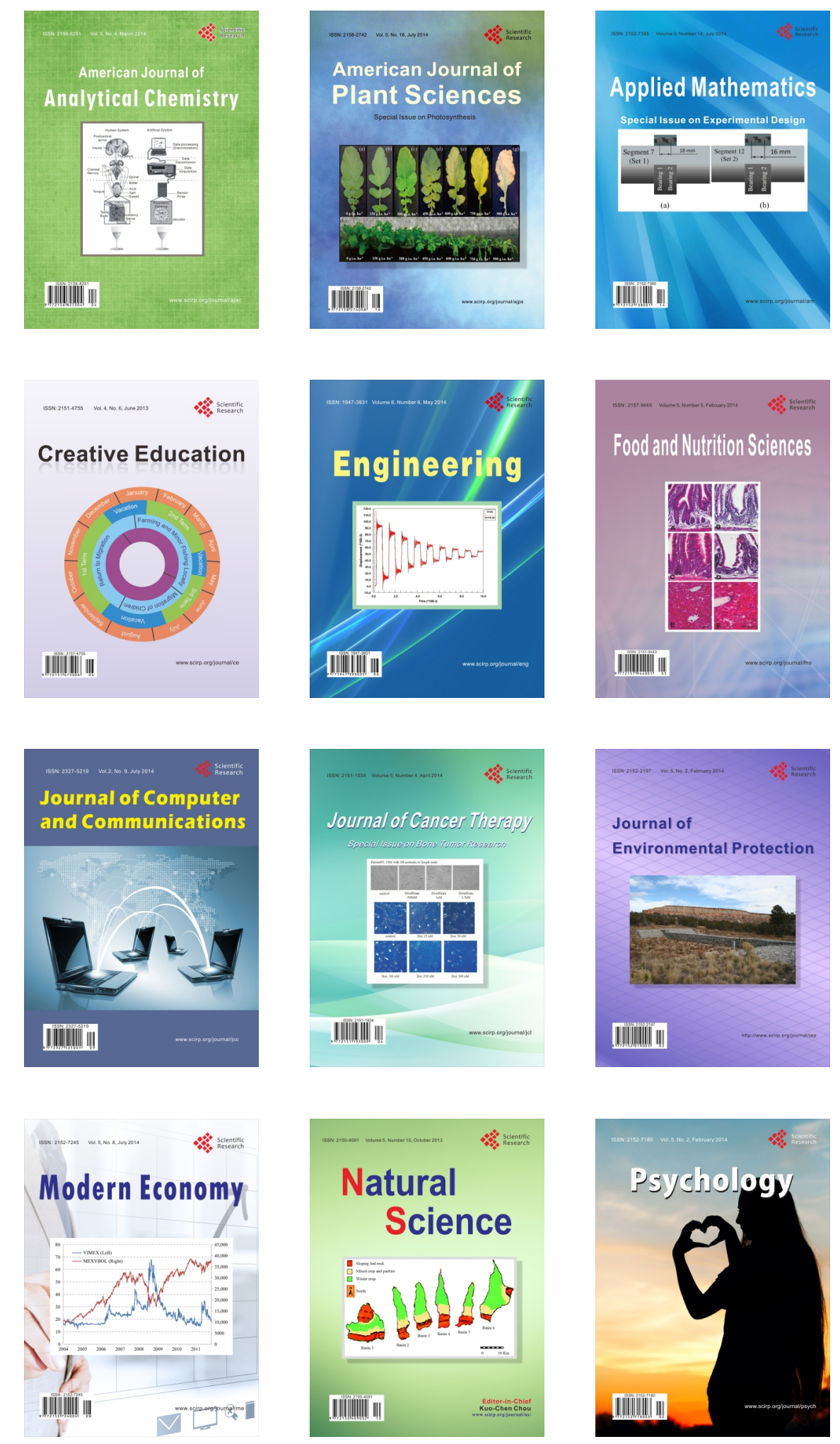